\title{
Percutaneous Modalities for Atrial Fibrillation Ablation
}

\author{
Tolga Aksu \\ Kocaeli Derince Education and Research Hospital, Department of Cardiology,Turkey \\ aksutolga@gmail.com
}

Pulmonary vein isolation (PVI) is now accepted as a cornerstone modality for the treatment of paroxysmal atrial fibrillation (PAF) ablation (1). The technique has been started with focal pulmonary vein (PV) ablation of AF triggers or ostial isolation of the PVs (2-5). Nowadays, wide circumferential ablation of the PV antrum is the preferred approach (6). The potential role of different non-PV targets for the treatment of PAF (either alone or as an adjunct to PVI) has been inconclusive, yet.

The description of PV as a trigger site of AF attracts leads to investigations that target focal ablation those sites within the PVs. However, the long-term results were unsatisfactory and the technique was associated with a significant risk of PV stenosis. So, segmental ablation at the anatomical ostium of the PVs was attempted in order to electrically isolate muscle sleeve connections between the PV and left atrium. Due to high recurrence ratios, the technique which initially targeted only the PVs with evidence of arrhythmogenic activity evolved electrically isolation of all PVs. To cope with PV stenosis, the ablation target was removed from PV ostium to the left atrial tissue defined as the PV antrum. Various three-dimensional electroanatomic mapping systems were used to achieve antral PV ablation $(7,8)$.

The recent trials showed that triggers exist not only within the PV but also outside the PV (e.g. superior vena cava, atrial septum, crista terminalis) (9). Once AF has been triggered, two mechanisms are responsible for sustaining the arrhythmia. One of them is multi-wavelet reentry (10). This theory is linked to the "critical mass" hypothesis of AF, whereby a certain atrial size is required to sustain enough wavelets for the arrhythmia. The second is the localized source model in which macroorganization in the form of spiral wave reentry (rotors) (11). According to the multi-wavelet hypothesis, the constant formation of new wavelets occurs through the process of wave splitting which occurs because of non-uniform dispersion of refractoriness and anatomic heterogeneity. This continuous propagation of multiple wavelets in the atria and wavelets as offspring of atrial reentry circuits might explain the mechanism by which AF is perpetuated without continuous focal discharge.

Multi-wavelet reentry hypothesis has caused to investigate some ablation modalities. One of them is ablation of complex fractionated atrial electrograms (CFAEs), which are thought to represent either continuous reentry of the fibrillation waves into the restricted area or an overlap of different wavelets entering the same area at different times (12). Ablation of these electrograms has been performed with the aim of eliminating wavelet reentry (13). Another approach is linear ablation lines within the left atrium which compartmentalizes the atria with the aim of preventing the formation of macro-reentrant circuits that have been postulated to maintain AF (14-16). Ablation lines include a roof line, a mitral isthmus line, and an anterior line.

Narayan et al. investigated the potential role to be sustained by localized sources such as electrical rotors and focal impulses (17). To identify rotor cores, focal impulse and rotor modulation mapping was developed using basket catheters by phase mapping. Rotors and focal sources identified by this approach are spatially constrained, temporally conserved, and drive AF based on maps of propagation and the ability of localized ablation at these sites to eliminate AF.

The autonomic system activity may play a role in the initiation and maintenance of AF, and modulating autonomic nerve function via ganglionated plexi (GP) ablation may increase the success of AF ablation. Ganglionated plexi sites may identify using continuous high frequency stimulation before antral PVI or circumferential PV ablation (18). 
Although PVI is usually enough for the success in PAF ablation, a stepwise ablation approach which consists of PVI, linear ablation, CFAE ablation, non-PV triggers ablation, and discretionary right atrial ablation are often necessary in persistent or long-standing persistent AF ablation. Isolation of PVs and posterior wall ablation are beginning steps, but identification and ablation of non-PV triggers or extensive substrate ablation are important to achieve long-term success.

\section{REFERENCES}

[1] Calkins H, Kuck KH, Cappato R, et al. 2012 HRS/EHRA/ECAS expert consensus statement on catheter and surgical ablation of atrial fibrillation: recommendations for patient selection, procedural techniques, patient management and follow-up, definitions, endpoints, and research trial design: a report of the Heart Rhythm Society (HRS) Task Force on Catheter and Surgical Ablation of Atrial Fibrillation. Developed in partnership with the European Heart Rhythm Association (EHRA), a registered branch of the European Society of Cardiology (ESC) and the European Cardiac Arrhythmia Society (ECAS); and in collaboration with the American College of Cardiology (ACC), American Heart Association (AHA), the Asia Pacific Heart Rhythm Society (APHRS), and the Society of Thoracic Surgeons (STS). Endorsed by the governing bodies of the American College of Cardiology Foundation, the American Heart Association, the European Cardiac Arrhythmia Society, the European Heart Rhythm Association, the Society of Thoracic Surgeons, the Asia Pacific Heart Rhythm Society, and the Heart

[2] Haïssaguerre M, Shah DC, Jaïs P, et al. Electrophysiological breakthroughs from the left atrium to the pulmonary veins. Circulation. 2000 Nov 14;102(20):2463-5.

[3] Marrouche NF, Martin DO, Wazni O, et al. Phased-array intracardiac echocardiography monitoring during pulmonary vein isolation in patients with atrial fibrillation: impact on outcome and complications. Circulation. 2003 Jun 3;107(21):2710-6.

[4] Verma A, Marrouche NF, Natale A. Pulmonary vein antrum isolation: intracardiac echocardiography-guided technique. J Cardiovasc Electrophysiol. 2004 Nov;15(11):1335-40.

[5] Chen SA, Hsieh MH, Tai CT, et al. Initiation of atrial fibrillation by ectopic beats originating from the pulmonary veins: electrophysiological characteristics, pharmacological responses, and effects of radiofrequency ablation. Circulation. 1999 Nov 2;100(18):1879-86.

[6] Khaykin Y, Skanes A, Champagne J, et al. A randomized controlled trial of the efficacy and safety of electroanatomic circumferential pulmonary vein ablation supplemented by ablation of complex fractionated atrial electrograms versus potential-guided pulmonary vein antrum isolation guided by intracardiac ultrasound. Circ Arrhythm Electrophysiol. 2009 Oct;2(5):481-7.

[7] Pappone C, Santinelli V, Manguso F, et al. Pulmonary vein denervation enhances long-term benefit after circumferential ablation for paroxysmal atrial fibrillation. Circulation. 2004 Jan 27;109(3):327-34.

[8] Kanagaratnam L, Tomassoni G, Schweikert R, et al. Empirical pulmonary vein isolation in patients with chronic atrial fibrillation using a three-dimensional nonfluoroscopic mapping system: long-term follow-up. Pacing Clin Electrophysiol. 2001 Dec;24(12):1774-9.

[9] Gerstenfeld EP, Callans DJ, Dixit S, Zado E, Marchlinski FE. Incidence and location of focal atrial fibrillation triggers in patients undergoing repeat pulmonary vein isolation: implications for ablation strategies. J Cardiovasc Electrophysiol. 2003 Jul;14(7):685-90.

[10] Moe GK, Abildskov JA. Atrial fibrillation as a self-sustaining arrhythmia independent of focal discharge. Am Heart J. 1959 Jul;58(1):59-70.

[11] Jalife, J, Berenfeld O, Skanes A, Mandapati R. Mechanisms of atrial fibrillation: mother rotors or multiple daughter wavelets, or both? J Cardiovasc Electrophysiol. 1998 Aug;9(8 Suppl):S2-12.

[12] Konings KT, Kirchhof CJ, Smeets JR, Wellens HJ, Penn OC, Allessie MA. High-density mapping of electrically induced atrial fibrillation in humans. Circulation. 1994 Apr;89(4):16658.

[13] Oral H, Chugh A, Good E, et al. Randomized evaluation of right atrial ablation after left atrial ablation of complex fractionated atrial electrograms for long-lasting persistent atrial fibrillation. Circ Arrhythm Electrophysiol. 2008 Apr;1(1):6-13.

[14] Hocini M, Jaïs P, Sanders P, et al. Techniques, evaluation, and consequences of linear block at the left atrial roof in paroxysmal atrial fibrillation: a prospective randomized study. Circulation. 2005 Dec 13;112(24):3688-96. 
[15] Jaïs P, Hocini M, Hsu LF, et al. Technique and results of linear ablation at the mitral isthmus. Circulation. 2004 Nov 9;110(19):2996-3002.

[16] Sanders P, Jaïs P, Hocini M, et al. Electrophysiologic and clinical consequences of linear catheter ablation to transect the anterior left atrium in patients with atrial fibrillation. Heart Rhythm. 2004 Jul;1(2):176-184.

[17] Narayan SM, Krummen DE, Shivkumar K, Clopton P, Rappel WJ, Miller JM. Treatment of atrial fibrillation by the ablation of localized sources: CONFIRM (Conventional Ablation for Atrial Fibrillation With or Without Focal Impulse and Rotor Modulation) trial. J Am Coll Cardiol. 2012 Aug 14;60(7):628-36.

[18] Verma A, Saliba WI, Lakkireddy D, et al. Vagal responses induced by endocardial left atrial autonomic ganglion stimulation before and after pulmonary vein antrum isolation for atrial fibrillation. Heart Rhythm. 2007 Sep;4(9):1177-82. 